\title{
We Asked the Experts: How Do We Maintain Surgical Quality Standards for Enhanced Recovery Programs After Cancer Surgery During the COVID-19 Outbreak?
}

\author{
Alexandre Doussot ${ }^{1} \cdot$ Bruno Heyd $^{1} \cdot$ Zaher Lakkis $^{1}$
}

Published online: 26 April 2020

(C) Société Internationale de Chirurgie 2020

Coronavirus Disease 2019 (COVID-19) is a novel viral disease that has spread rapidly worldwide, becoming a pandemic. Among infected patients, around 20-30\% of patients will require intensive care unit (ICU) admission due to acute respiratory distress syndrome [1]. Consequently, the health care workforce allocation has been widely disrupted at an institutional level in an attempt to manage the surge of COVID-19 patients requiring ICU care. While the workload has increased in all institutions fighting the outbreak, intensivists and ICU nurses are at risk of physical and mental exhaustion. Consequently, anesthesiologists and non-ICU nurses are being re-assigned to serve as in intensive care settings. As part of the general effort, elective surgical caseload has been substantially decreased to allow for better ICU and hospital bed capacity. In this setting, surgical procedures should be restricted to life threatening conditions requiring emergent surgical management.

Regarding cancer surgery, most procedures have been postponed. Due to the potential negative impact of treatment delays on prognosis, surgical, and oncological societies have published guidelines to identify ideal clinical situations when semi-elective cancer surgery can proceed, and have suggested alternative management strategies to the standards of care in clinical situations where primary surgical treatment cannot be undertaken [2, 3].

Maintaining surgical quality standards, such as enhanced recovery programs (ERP) after cancer surgery, is of paramount importance to assure that cancer patients in-

Alexandre Doussot

adoussot@chu-besancon.fr; lex.doussot@yahoo.com

1 Department of Digestive Surgical Oncology - Liver

Transplantation Unit, University Hospital of Besançon, 2, Boulevard Alexander Fleming, 25000 Besançon, France hospital stays are minimized, as inpatient resources need to be redirected to COVID-19 patients. However, when facing organizational disruption due to the COVID-19 outbreak, adherence to ERP after surgery can be challenging. ERPs rely on dedicated multidisciplinary teams including nurses, anesthesiologists, and surgeons who ensure program compliance for improved outcomes. Any disruption in this teamwork may result in decreased compliance, leading to variation in care, which, in turn, can impair postoperative outcomes. Due to the outbreak, aside from the risk of contracting COVID-19 infection, ERP members often become focused on other objectives out of necessity.

First, due to re-assignment to another unit, ERP nurses in charge of preoperative patient education and postoperative monitoring and compliance might be unavailable. Second, in the absence of dedicated ERP anesthesiologists, non-ERP anesthesiologists assigned to cancer surgery cases might be unaware of ERP guidelines [4, 5]. Consequently, intraoperative goal-directed fluid therapy and opioid-free anesthesia, both advocated by ERP guidelines, may not be applied adequately and uniformly. Additionally, using steroids for preventing postoperative nausea and vomiting or non-steroidal anti-inflammatory drugs for pain control remains disputed in the current setting. Anti-inflammatory drugs, especially non-steroidal anti-inflammatory drugs, have indeed been possibly associated with more severe COVID-19 infection. To date, guidelines for the perioperative use of anti-inflammatory drugs in the setting of COVID-19 are lacking. Third, from a surgical standpoint, minimally invasive surgery is fully integrated into ERP guidelines, yet, as scientific knowledge about COVID-19 is growing daily, the risk of contracting COVID-19 due to aerosol exposure during laparoscopic or thoracoscopic surgery remains a possible concern. In this context, surgical societies have made recommendations for prevention of 
laparoscopic aerosolization. Based on these, institutional policies should be established and enforced regardless of the patient's COVID-19 status. Finally, as we are all living in these difficult times with significant pressures and concerns, patients' anxiety could hamper their adherence to the ERP recommendations. Taken altogether, these constraints may impede compliance to the protocol and question the safety of applying ERP by non-dedicated teams.

It is the responsibility of the institutions and the medical staff to reassure patients by creating safe postoperative surgical wards and environments free of COVID-19 as much as possible. Thus, preoperative screening of all patients for COVID-19 is paramount. In the event of a positive COVID-19 infection, elective cancer surgery must be postponed, as the risk of postoperative complications and possibly death may outweigh any survival benefit from surgery [6].

In France, the nationwide emergency plan to fight the outbreak started on March 13th. Regional health agencies asked all medical institutions to cancel non-emergent surgical activity. In our tertiary care referral center, while emergency surgery is ongoing, the elective cancer surgery caseload has been drastically reduced from 24 to 4 operative rooms per day. Very few cancer surgery procedures remain scheduled upon validation at a daily multidisciplinary surgical meeting involving multiple surgical specialists such as surgical oncologists, urologists, orthopedists, and neurosurgeons among others. Regarding our digestive surgical oncology unit, this translates in a decrease from 10 to 15 cancer surgery cases before the outbreak to around 3 a week over the last 3 weeks.

In order to overcome the issues listed above, especially regarding staffing issues, the following adaptations have been made to maintain ERP: as our two ERP nurses have been re-assigned to other units, ERP surgeons are responsible for preoperative patient information and education; regarding intraoperative compliance, all ERP anesthesiology protocols have been made available by the ERP-dedicated anesthesiologists to guide those anesthesiologists who are not familiar with ERP; additionally, although our six ERP-dedicated anesthesiologists have been re-assigned to the COVID-19 ICU, one remains available for most of our elective cases; from a surgical standpoint, while half of our surgical team rotates every 2 weeks, standard surgical principles have not changed, and we continue to follow the no-drain, no-gastric tube policy, as recommended by ERP guidelines; to maximize the safety of laparoscopic surgery, we systematically use gas extraction devices throughout each procedure; regarding postoperative care and monitoring, nurses from our inpatient unit are familiar with ERP items, and they encourage patients for early mobilization and eating; finally, while in person monthly meetings for quality assessment are canceled due to social distancing, quality, and safety control need to be continued through video-conferencing and online newsletters.

It remains to be determined whether the current outbreak will negatively impact the compliance with ERP. Nevertheless, we do believe that ERP after cancer surgery should be maintained as much as possible, provided its safety is ensured. Further, as the peak of the outbreak might soon be reached, strained health care resources could persist overtime, and all systems worldwide will remain firmly impacted. As a result, the aforementioned issues might be encountered for months to come. Updating guidelines to help tailor ERP to the current situation could be of value.

\section{References}

1. Onder G, Rezza G, Brusaferro S (2020) Case-fatality rate and characteristics of patients dying in relation to COVID-19 in Italy. JAMA. https://doi.org/10.1001/jama.2020.4683

2. Tuech JJ, Gangloff A, Di Fiore F et al (2020) Strategy for the practice of digestive and oncological surgery during the Covid-19 epidemic. J Visc Surg. S1878-7886(20):30070-30079. https://doi. org/10.1016/j.jviscsurg.2020.03.008

3. Ueda M, Martins R, Hendrie PC et al (2020) Managing cancer care during the COVID-19 pandemic: agility and collaboration toward a common goal. J Natl Compr Canc Netw. https://doi.org/10.6004/ jnccn. 2020.7560

4. Gustafsson UO, Scott MJ, Hubner M et al (2019) Guidelines for perioperative care in elective colorectal surgery: enhanced recovery after surgery $\left(\right.$ ERAS $\left.^{\circledR}\right)$ society recommendations: 2018 . World J Surg 43:659-695. https://doi.org/10.1007/s00268-018-4844-y

5. Melloul E, Hübner M, Scott M et al (2016) Guidelines for perioperative care for liver surgery: enhanced recovery after surgery (ERAS) society recommendations. World J Surg 40:2425-2440. https://doi.org/10.1007/s00268-016-3700-1

6. Aminian A, Safari S, Razeghian-Jahromi A et al (2020) COVID19 outbreak and surgical practice: unexpected fatality in perioperative period. Ann Surg. https://doi.org/10.1097/SLA. 0000000000003925

Publisher's Note Springer Nature remains neutral with regard to jurisdictional claims in published maps and institutional affiliations. 